\title{
Review: ELISA D-dimer is sensitive but not specific in diagnosing pulmonary embolism in an ambulatory clinical setting
}

Brown MD, Rowe BH, Reeves MJ, et al. The accuracy of the enzyme-linked immunosorbent assay D-dimer test in the

diagnosis of pulmonary embolism: a meta-analysis. Ann Emerg Med 2002;40:133-44.

\section{QUESTION: What is the accuracy of the enzyme linked immunosorbent assay (ELISA) $\mathrm{D}$-dimer test in diagnosing pulmonary embolism (PE) in the emergency department or outpatient clinic?}

\section{Data sources}

Studies were identified by searching Medline (January 1980 to 1 January 2001), EMBASE/Excerpta Medica, and the bibliographies of previous systematic reviews and identified studies; and by contacting experts in the field and the manufacturers of ELISA D-dimer.

\section{Study selection}

Studies were selected if they prospectively investigated the use of ELISA D-dimer tests in patients with suspected PE who presented to an emergency department or outpatient clinic. Reviews and editorials were excluded.

\section{Data extraction}

Data were extracted independently by 2 reviewers on study quality, patient characteristics, the diagnostic standard, and the sensitivity and specificity of the test.

\section{Main results}

11 studies involving 2126 patients met the selection criteria. The prevalence of $\mathrm{PE}$ ranged from $17-58 \%$. The mean age range of patients was 54-81 years. Receiver operating characteristic curve analysis found significant heterogeneity among the studies. Pooling the results from the 11 studies using a random effects model resulted in high sensitivity and low specificity (table). Subgroup analysis of the 9 studies that used traditional ELISA D-dimer methods (ie, not rapid ELISA methods) yielded similar results (table). Advanced patient age $(\geq 70 \mathrm{y})$ resulted in lower specificity $(14 \%)$. A prolonged duration of symptoms ( $\geq 3 \mathrm{~d}$ ) decreased both sensitivity and specificity (1 study, $73 \%$ and $33 \%$, respectively).

\section{Conclusion}

The enzyme linked immunosorbent assay D-dimer test is highly sensitive but not specific in diagnosing pulmonary embolism in an ambulatory clinical setting.

Source of funding: no external funding.

For correspondence: Dr M D Brown,

Spectrum Health-Butterworth, Grand Rapids, Michigan, USA. brownm@msu.edu

Abstract and commentary also published in $A C P$ Journal Club

Test properties of enzyme linked immunosorbent assay D-dimer for pulmonary embolism in an ambulatory clinical setting*

\begin{tabular}{cllll} 
Number of studies & Sensitivity $(95 \%$ Cl) & Specificity (CI) & +LR & -LR \\
\hline 11 & $95 \%(90$ to 98$)$ & $45 \%(38$ to 52$)$ & 1.73 & 0.11 \\
\hline 9 & $94 \%(88$ to 97$)$ & $45 \%(36$ to 55$)$ & 1.71 & 0.13 \\
\hline
\end{tabular}

*Diagnostic terms defined in glossary; LRs calculated using the pooled summary estimates of sensitivity and specificity reported by the author.

\section{COMMENTARY}

Based on this sound meta-analysis by Brown $e t$ al, the role of D-dimer measurement as a diagnostic aid in patients with clinically suspected PE is confirmed. As initially suggested $>10$ years ago, ${ }^{1}$ a D-dimer level below a certain cut point rules out $\mathrm{PE}$ with a high predictive value, at least in patients with a low or moderate clinical probability.

Why is D-dimer useful in ruling out PE? Why not use diagnostic tests that are both sensitive and specific, such as pulmonary angiography or spiral computed tomography (CT)? The main reason is that the index of suspicion for PE has increased over the years, lowering its confirmed prevalence to $\leq 20 \%$ in clinically suspected populations. Thus, ruling out pulmonary embolism with a noninvasive, quick, and relatively inexpensive test has become a priority. Secondly, angiography and spiral CT are costly. Thus, sparing them in patients with a low D-dimer level should be cost effective, although this has not been shown formally.

Some limitations of D-dimer testing deserve attention. Firstly, it is not a stand alone test, and should be used as part of a diagnostic algorithm, which includes a clinical probability assessment. ${ }^{2}$ Secondly, it is most useful in outpatients because certain comorbid conditions in hospital patients can increase D-dimer levels. Thirdly, this meta-analysis was restricted to studies that used ELISAs; recent studies suggest that some automated latex assays may also be highly sensitive to venous thromboembolism while other latex assays are not sensitive enough to rule out PE unless patients have a low clinical probability.

Considering these limitations, should the results of D-dimer testing be used to help determine the management of patients with suspected PE? According to a published clinical management study, ${ }^{3}$ yes. However, further studies are needed to confirm that a negative D-dimer test result, when combined with a low or moderate clinical likelihood for PE, is associated with a low rate of thromboembolism that is similar to that seen with an established diagnostic standard, such as a normal ventilation perfusion lung scan. ${ }^{4}$

Henri Bounameaux, MD University Hospital of Geneva Geneva, Switzerland

Bounameaux H, Cirafici P, de Moerloose P, et al. Measurement of D-dimer in plasma as diagnostic aid in suspected pulmonary embolism. Lancet 1991;337:196-200. Perrier A, Bounameaux H. Cost-effective diagnosis of deep vein thrombosis and pulmonary embolism. Thromb Haemost 2001;86:475-87.

Perrier A, Desmarais S, Miron MJ, et al. Non-invasive diagnosis of venous thromboembolism in outpatients. Lancet 1999;353:190-5.

Hull RD, Raskob GE, Coates G, et al. Clinical validity of a normal perfusion lung scan in patients with suspected pulmonary embolism. Chest 1990;97:23-6. 